mation in the insulator in the form of trapped charge. The device is simple, very small, and ideally suited for fabrication in large integrated arrays.

The device described by HolmKennedy and Heald is based, surprisingly, on a simple semiconductor diode or transistor structure and is the first to employ a phenomenon known as field-induced trapping. The charge state of certain impurities in the lattice of a semiconductor can be changed by applying a strong field which allows carriers to surmount a potential barrier surrounding the impurity that is caused by the existing Coulombic charge on the impurity. The carrier can remain trapped for some time even after the field is removed. In its altered state, the impurity has an increased scattering effect on carriers, so the result is a differential negative mobility effect which may be slow to decay.

Kroemer (Phys. Rev., 109, 1856; 1958), Ridley and Watkins (J. Phys. Chem. Solids, 22, $155 ; 1961)$ and Hilsum (Proc. Inst. Radio Eng., 50, 185; 1962) proposed that negative mobility characteristics should be used to produce microwave oscillations. In fact, Gunn independently happened upon such oscillations in gallium arsenide in 1963 (Solid State Commun., 1, 88). The negative mobility in this case is not due to field-induced trapping, but to transferred electron effects. The field-induced trapping effect was demonstrated by Ridley and Pratt in germanium doped with gold, and found to be too slow for microwave purposes (J. Phys. Chem. Solids, 26, 21 ; 1965).

Holm-Kennedy realized that this slowness of trap population and depopulation could be useful. For certain defects it might be possible to reduce mobility for long periods but in a reversible manner-in other words to control the "dumping" of charge in and out of deep-lying impurity centres in semiconductors-and to use this as the basis of a memory device. Copper in silicon seemed a suitable system and, on the first attempt, HolmKennedy and a colleague produced a diode with memory action. The memory effect had a long life and the diode could be left unconnected for weeks in the altered state without significant change. A similar change can also be made in a transistor structure, which increases the range of circuits in which the principle can be made use of. Furthermore, the diode device was multistable (the degree of alteration could be graded), unlike the bistable flipflop.

As the change of state requires times of the order of milliseconds, the principle may be slow for some memory applications-flip-flops can switch in a few nanoseconds-and it remains to be seen whether the trapping and de- trapping process can be repeated many millions of times without fatigue, a necessary feature of a computer memory. If no fatigue is evident, this storage principle will constitute a definite improvement over the MIS device.

This latest invention is one of the first uses of a subtle feature of deep-lying defect energy levels in semiconductors. Until now, defects have been regarded as damage, to be minimized or, alternatively, to be used as a blunt instrument to "kill" some unwanted property in a material. This invention points to the possibility of a new generation of devices which use the more subtle properties of defects. Indeed, the point is not being ignored by those who have to do with electronic devices, for, in two conferences this summer, there has been discussion of the use of other types of defect introduction to enhance diffusion and modify carrier lifetimes. The recent increase in basic knowledge about dislocations and the effects of particle bombardment now seems to be percolating through to the benefit of the semiconductor technologist.

\section{SYNAPSE \\ Resolution of Proteins}

from our Neurochemistry Correspondent RECENT rapid advances in the isolation of pharmacological receptors have revealed that synaptic events can be analysed at a molecular level, and have suggested that individual protein, or protein-containing, molecules occupying discrete areas of the surface membrane at the synapse-or elsewhere-may subserve specific functions. The synapse itself is specialized for the transfer of information between nerve cells by chemical transmitters, and receptors, as well as enzymes or carrier sites for the inactivation of transmitters, are clearly part of the molecular equipment developed for carrying out this particular task.

The complex integrative actions of the nervous system, including highly accurate recognition of sites for contact during development, imply, however, further specializations at the synapse, which may require their own particular molecular machinery. As yet little is known about such aspects of synaptic function, but one possible approach to the problem is to make an overall chemical analysis of the synapse with the object of isolating and characterizing any other molecules specific to this region, and hence, possibly, determining their function. A recent paper by McBride and VanTassel (Brain Res., 44, $177 ; 1972$ ) describing a protein analysis of components in nerve endings is therefore an encouraging start.

One of the obstacles to performing a meaningful chemical analysis so far has been the difficulty of obtaining a reasonably pure preparation of starting material. The subcellular fractionation methods of Whittaker and others allow the separation from brain homogenates of fractions relatively enriched in nerve endings - "synaptosomes" - using

\title{
Measuring Schwarzschild Coordinates
}

Many elegant tests of relativistic properties of massive objects exist only in the mind, as gedanken experiments; equally, all too often practical tests of such parameters involve laborious statistical work or a process of elimination in which all non-relativistic effects are explained away and a tiny residue of otherwise unexplained perturbation is pulled out of the hat as the relativistic effect. Both processes have their faults. In next Monday's Nature Physical Science (October 30), however, Banerji describes how an observer in the exterior gravitational field of a star can find whether he is at rest in Schwarzschild coordinates by a technique which is both practicable and elegant.

Banerji does, of course, make some assumptions; the star must be nonrotating, have constant radius, and produce a spectrum including discrete lines. But all these requirements are less drastic than the assumption of weak fields which other students of this problem have made and which Banerji does not. It is reasonable, according to Banerji, to make these assumptions for the Sun, neglecting rotation and the influence of the planets, so his experiment could be carried out from a test vehicle in the solar system.

An observer on board such a vehicle can determine the local standard of rest first by adjusting his motion so that he sees a constant redshift in the spectra from the star; this ensures no acceleration. To check that there is no constant velocity component producing a Doppler shift in the spectra Banerji suggests studying the orbits of projectiles launched from the test vehicle, and he gives the equations necessary to deduce when the vehicle is indeed at rest relative to the star's coordinate frame. If two observers in two such vehicles carry out the same experiments, it is a simple matter for them to determine, from their measurements at different constant positions relative to that frame, the Schwarzschild mass and the radius of the underlying star. As Banerji says, "a high precision in rocket control and measurement of the redshift must be achieved"; but at least the experiment would work in practice. 